\title{
Major Salivary Gland Adenocarcinoma
}

National Cancer Institute

\section{Source}

National Cancer Institute. Major Salivary Gland Adenocarcinoma. NCI Thesaurus. Code C5903.

An adenocarcinoma that arises from the parotid gland, submandibular gland, or sublingual gland. 